\title{
Molecular Cloning, Expression Pattern, and Phylogenetic Analysis of the Lysyl-tRNA Synthetase Gene from the Chinese Oak Silkworm Antheraea pernyi
}

\author{
Yan-Qun Liu and Li Qin \\ Department of Sericulture, College of Bioscience and Biotechnology, \\ Shenyang Agricultural University
}

China

\section{Introduction}

Aminoacyl-tRNA synthetases (AARS) are a class of enzymes that charge tRNAs with their cognate amino acids. There are two classes of tRNA synthetases, classes I and classes II, that are distinguished by the architectures of their active-site catalytic cores (Guo et al., 2008). Lysyl-tRNA synthetase (LysRS) is an AARS, a group of ancient proteins known for their critical role in translation. LysRS is a homodimer localized to the cytoplasm which belongs to the class II family of tRNA synthetases. Its assignment to class II AARS is based upon its structure and the presence of three characteristic sequence motifs in the core domain. The catalytic core domain is primarily responsible for the ATP-dependent formation of the enzyme bound aminoacyl-adenylate.

The LysRS gene has been cloned and characterized from various kinds of organisms. In Escherichia coli, there are two distinct LysRSs encoded by two widely separated genes, LysRS and LysRU (VanBogelen et al., 1983; Emmerich \& Hirshfield, 1987), while in Campylobacter jejuni only one LysRS is present (Chan \& Bingham, 1992). In E. coli, the LysRS gene is expressed constitutively while LysRU can be induced by growth at high temperature (Hirshfield et al., 1981). In human, only one LysRS belonging to the class II family of AARSs is present (Guo et al., 2008). The crystal structure of tetrameric form of human LysRS has been recently determined (Guo et al., 2008). Human LysRS has been shown to be secreted and to trigger a proinflammatory response as a target of autoantibodies in the human autoimmune diseases, polymyositis or dermatomyositis (Park et al., 2005). And, LysRS is required for the translocation of calreticulin to the cell surface in immunogenic death (Kepp et al., 2010). However, none of insect LysRS gene has been characterized to date.

The Chinese oak silkworm, Antheraea pernyi (Lepidoptera: Saturniidae), is one of the most well-known economic insect species used for silk production and insect food source. This insect is known to be sucessfully domesticated in China around the 16th century (Liu et al. 2010a), and it is commercially cultivated in China, India, and Korea. To isolate the functional genes of A. pernyi, we have constructed a full-length cDNA library (Li et al. 2009). By EST 
sequencing, several A. pernyi genes encoding important enzymes have been characterized, such as two enolase genes and a lysophospholipase gene (Liu et al. 2010b, 2010c).

In this chapter, we describe the cloning and characterization of the A. pernyi LysRS gene from the full-length pupal cDNA library by random EST sequencing. The expression patterns at various developmental stages and different tissues were investigated. Finally, the deduced protein sequence of the LysRS gene from A. pernyi and other organisms were used to examine the relationship among these species, and to test the potential use of LysRS protein in phylogenetic study.

\section{Materials and methods}

\subsection{Silkworms and tissues}

The A. pernyi strain Shenhuang No. 1 was used in this study. Larvae were reared routinely on oak trees (Quercus liaotungensis) in the field. Blood, fat body, midgut, silk glands, body wall, Malpighian tubules, spermaries, ovaries, brain and muscle were taken from silkworm larvae at day 10 of fifth instar. Eggs at day 5, larvae at day 10 of fifth instar, pupae and moths were also sampled. All the samples were immediately frozen in liquid nitrogen and stored at $80^{\circ} \mathrm{C}$ for later use.

\subsection{Cloning of the A. pernyi LysRS gene}

A full-length pupal cDNA library of $A$. pernyi has been constructed in our lab (Li et al. 2009). An EST encoding LysRS homolog (GenBank accession no. GH335029) was isolated by random EST sequencing. So, the cDNA clone was used to complete the full-length cDNA sequence of the $A$. pernyi LysRS gene.

\subsection{Sequence analysis}

DNASTAR software (DNASTAR Inc., Madison, WI) was used to identify open reading frame (ORF), deduce amino acid sequence, and predict the isoelectric point and molecular weight of the deduced amino acid sequence. Blast search was performed at http://www.ncbi.nlm.nih.gov/blast/. Conserved Domains was predicted at http://www.ncbi.nlm.nih.gov/Structure/cdd/wrpsb.cgi/. The in silico gene expression analysis based on the available EST resources was employed at http://www.ncbi.nlm.nih.gov/Unigene/ESTprofileViewer/.

\subsection{Total RNA extraction and first strand cDNA synthesis}

Total RNA was extracted by using RNAsimple Total RNA Extraction Kit (TIANGEN Biotech Co. Ltd., Beijing) according to the manufacturer's instruction. DNase I was used to remove contaminating genomic DNA. The purity and quantity of the extracted RNA was quantified by the ratio of OD260/OD280 by ultraviolet spectrometer. First strand cDNA was generated by using $2 \mu \mathrm{g}$ of total RNA per sample with TIANScript cDNA Synthesize Kit (TIANGEN Biotech Co. Ltd., Beijing).

\subsection{RT-PCR analyses}

The cDNA samples were amplified by semi-quantitative PCR method using the genespecific primer pair LYQ146 (5'-TCCGA GTGGG GAAGA AGTTG-3') and LYQ147 (5'TTCAG TCAGT CCTGG TATGT-3') for the A. pernyi LysRS gene, which generated a 322 bp 
fragment. An actin gene (GU073316) was used as an internal control, and a $468 \mathrm{bp}$ fragment was amplified using the primer pair LYQ85 (5'-CCAAA GGCCA ACAGA GAGAA GA 3') and LYQ86 (5'CAAGA ATGAG GGCTG GAAGA GA 3') (Wu et al, 2010). PCRs were performed with the following cycles: initial denaturation at $95^{\circ} \mathrm{C}$ for $5 \mathrm{~min}$; followed by 30 cycles of $1 \mathrm{~min}$ at $95^{\circ} \mathrm{C}, 30 \mathrm{~s}$ annealing at $55^{\circ} \mathrm{C}, 30 \mathrm{~s}$ extension at $72^{\circ} \mathrm{C}$; and a final extension at $72^{\circ} \mathrm{C}$ for $10 \mathrm{~min}$. To avoid sample DNA contamination, the negative RT-PCRs control reactions were performed with every total RNA as templates. The amplification products were analyzed on $1.0 \%$ agarose gels, purified from the gel, and directly sequenced to confirm the specificity.

\subsection{Phylogenetic analysis}

The amino acid sequences of LysRS homologs from different organisms were retrieved from GenBank database, and SilkDB database (Duan et al., 2010). Multiple sequence alignments were performed using Clustal X software (Thompson et al. 1997). A phylogenetic tree was constructed by MEGA version 4 (Tamura et al. 2007) using Neighbour-Joining (NJ) method (Saitou and Nei 1987) with bootstrap test of 1000 replications.

\section{Results and discussion}

\subsection{Sequence analysis of the A. pernyi LysRS gene}

We identified the A. pernyi LysRS gene from a pupal cDNA library constructed in our lab by random EST sequencing (Li et al., 2009). Based on the cDNA clone Appu0107, we isolated and sequenced a full-length cDNA of the A. pernyi LysRS gene. The cDNA sequence and deduced amino acid sequence of the A. pernyi LysRS gene were shown in Figure 1. The obtained 2136 bp cDNA sequence contains a 5'-untranslated region (UTR) of $70 \mathrm{bp}$, a 3' UTR of $292 \mathrm{bp}$ with a polyadenylation signal sequence AATAAA at position 2082 and a poly (A) tail, and an ORF of 1740 bp encoding a polypeptide of 579 amino acids. The LysRS protein has a predicted molecular weight of $65.62 \mathrm{kDa}$ and isolectric point of 6.1. Blast search revealed that the deduced amino acid sequence of the A. pernyi LysRS gene had $66 \%$ identities and $79 \%$ positives with that of Homo sapiens LysRS (NP_005539), which belongs to the class II family of AARSs (Guo et al., 2008). Conserved Domains prediction showed that the A. pernyi LysRS protein contained the LysRS class II core domain (Wolf et al., 1999), including the conserved active sites and three characteristic sequence motifs (Desogus et al., 2000). Moreover, the characteristic signature HIGH sequence of LysRS class I was not present. We therefore referred to the protein as LysRS of $A$. pernyi. This cDNA sequence has been deposited in GenBank under accession no. JF773568.

\subsection{Homologous alignment}

By searching in database, the A. pernyi LysRS protein homologues were found in various kinds of life organisms, including bacteria, fungi, plants, invertebrates and vertebrates. To assess the relatedness of A. pernyi LysRS to LysRS proteins from other organisms, identities were calculated based on a Clustal alignment including 43 representative LysRS protein sequences (Figures 2 and 3). The other 42 LysRS protein or homologue sequences used in this study were downloaded from GenBank database, with one exception of B. mori LsyRS from SilkDB database (Duan et al., 2010). These protein sequences were from 3 bacteria, 1 fungi, 6 plants, 22 invertebrates and 11 vertebrates. By sequence alignment, the $A$. pernyi 
71 ATGCCAGAACCTOCAGTGAAAA AGTT TCAAAGAATGAGTT AA AGOGOCGGTTAA AACC TGAACAGA AATT AAAGGAGA AA

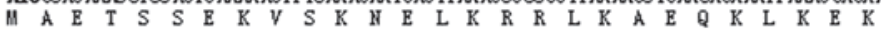

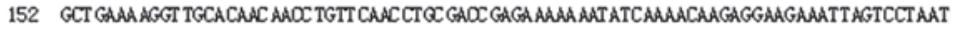
A $\underset{B}{\mathbb{R}} \mathrm{K}$ V

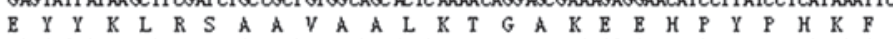
314 ACT GTGACAAT TTCT TT AGAAGAATTC ATTAATAAGT AC AATAATTT AAATAGTGGAGAGTGCTAGAGATT ACAACTGTA

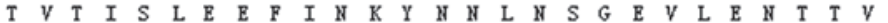
395 TCACTOGCT GQCGATC ATTC TATT AGGGAGTCOGGA QCTAAACT AATATTCTATGACTTGAGAGCTGAOGAGTGAAA

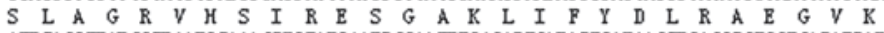

476 ATT CAGGTTAT GGCT AATGCAAACTTGTATGAATCGAATTTGAGAC TGATACTGATAGCTGAOGCGTGGTGACATCATA

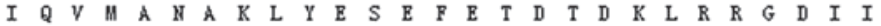

557 GGT TGCATT GGACACOAGGAAA AACA AAGA AAOGQGAGTTGTOAT TGTACCACAATCAATTAAGTTACTCTCTCCATGT

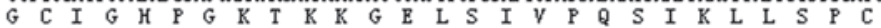

638 CTT CATA TGCT $Q C C T$ CATT TACACTTT QGTC TCAAAGAT AAA AANCOOGTTTAGGAGGAGATATTT AGATCTCATTTTA L

719 ANT GATC AGGT AHGGCAAA CATT CTAT ACAAGACC CAHANTTATACC ATATGTAAGAGATTTTTAGATAATATGGOCTTC

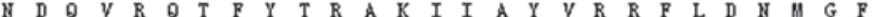

ஜ0 TTAGAGA TAGAAOCOCAA TGAT GALCATGA TCO AGGA GGAGCT AC ACAAHACCTTTATAACACATCACAAT GATCTT $\begin{array}{llllllllllllllllllllllllllll}L & E & I & E & T & P & M & \| & \mathbb{N} & M & I & P & G & G & A & T & A & K & P & F & I & T & H & H & \mathbb{H} & D & L\end{array}$

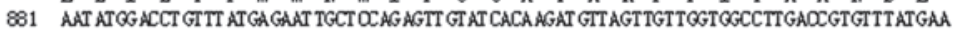

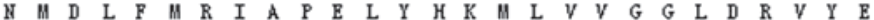

962 ATT GGAOGCAGTTCOGTAATGAGGT ATAGATTT AACACACAACO TGAATTTACAACATGTGALTTTTATATGGCATAC $\begin{array}{llllllllllllllllllllllllllll}I & G & R & Q & F & R & \mathbb{N} & E & G & I & D & L & T & H & \mathbb{A} & P & E & F & T & T & C & E & F & Y & M & \text { A } & Y\end{array}$ 1043 GCT GATT HCAATGATCTAATT AC TATA ACAGAAN AATGTT GT CAOGAATGGTGAAAACATTCATGGTACTTATAAGGTA

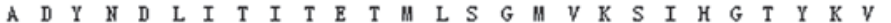
1124 AAATAOCATO AGAT OGTCOGAGTGOGGAAGAGT TGAAATTGATTT TACTCCOCCATTTOCTAGAGTTCCTATGATTGCG

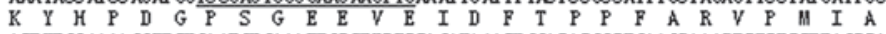

1206 ACT TTGGAAAQGTTCT GA AT GT GAAATTCCT TC TCCAGATAAACT GGACACOGCTGAACCAAACTCTCTCCTTAOCCAA

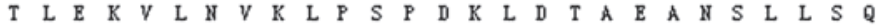

1286 TTA TGTGAAAAACAT GAOG TGGAATOCOACCAOC QCGGACAACAOC TAGACT ACTTGATALATTAGTTGGTGAATTCCTT $\begin{array}{llllllllllllllllllllllllllll}L & C & E & K & H & E & V & E & C & P & P & P & R & T & T & A & R & L & L & D & K & L & V & G & E & F & L\end{array}$

1367 GAAGACAAATGCATC AAOC CAA ATTT ATTC TGGATCATOCAC AAAT TAT GAGOCCATT GTCTAAGTATCACAGGGACATA

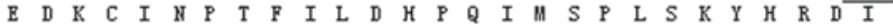

1448 CCA GGACTGAC TGAAAGAT TT GAACTA TTOGTAAT GAAGAHGAAATCTGTAATGCGIATACT GAGTTAAATGACCCTGCT $\begin{array}{llllllllllllllllllllllllllll}P & G & L & T & E & R & F & E & L & F & Y & M & K & K & E & I & C & N & A & Y & T & E & L & \mathbb{N} & D & P & A\end{array}$ 1529 ACT CAGA GAGAAGGTTTGAGCA ACAACTAAAGA TCGA QCAGCT GGTGATGATGAGACTOCACOGACAGATGAAGCATTC

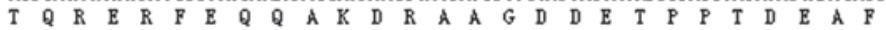
1610 TGT ACTGOCT OGAATATGGACT TCCAOCCACT C TGGT TGOGGACT TOGCGTOGATOGCCTCACT AT GTTTTTAACAGAT

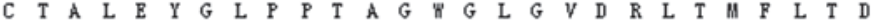
1691 GCAAACANCAT TAAG GAAGTATT OCTT TTOCT C AATGAACCAGA TGATCCGALCAAGCATANAATGAAGAGGGAAT A $\mathbb{N}$ \& 1772 GCAQCOGATAGCACT OCCT TATT ACAAAATGGACT TAGICAATGAGACA GT GT TCACAATT AACACT GT TAAAAGTT TT A A D S T P L L Q II G A *

1853 TCACATA GAAAAGTT TT AC TANC ALAT TTCA TAAC TATT CAOC TCCT ACTCAT ACTTAACOCTTTGCACTCGTAACTTTTT 1934 TCATTTG TATT ACCA CCAGCT AGAAAC TADGATTGTTTGCT GT TATC GAGAGCATAACAAOCTGTGACAGTCATTT GTCCC

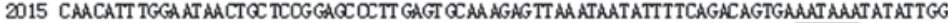

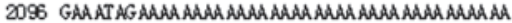

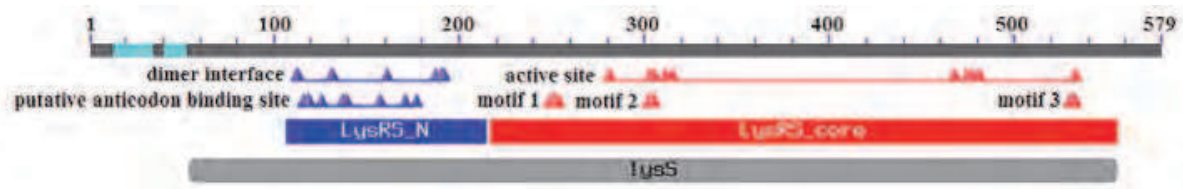

Fig. 1. The complete nucleotide and deduced amino acid sequence of the A. pernyi LysRS gene. (A) cDNA sequence and deduced amino acid sequence of the LysRS gene. The amino acid residues are represented by one-letter symbols. The initiation codon ATG is bolded and the termination codon TAG is bolded and marked with an asterisk. The polyadenylation signals AATAAA are double-underlined. The gene specific primer sequences used in the semi-quantitative RT-PCR experiment are underlined. The cDNA sequence was deposited in GenBank under accession no. JF773568. (B) Conserved domains of A. pernyi LysRS determined by http://www.ncbi.nlm.nih.gov/Structure/cdd/wrpsb.cgi/. 
Molecular Cloning, Expression Pattern, and Phylogenetic

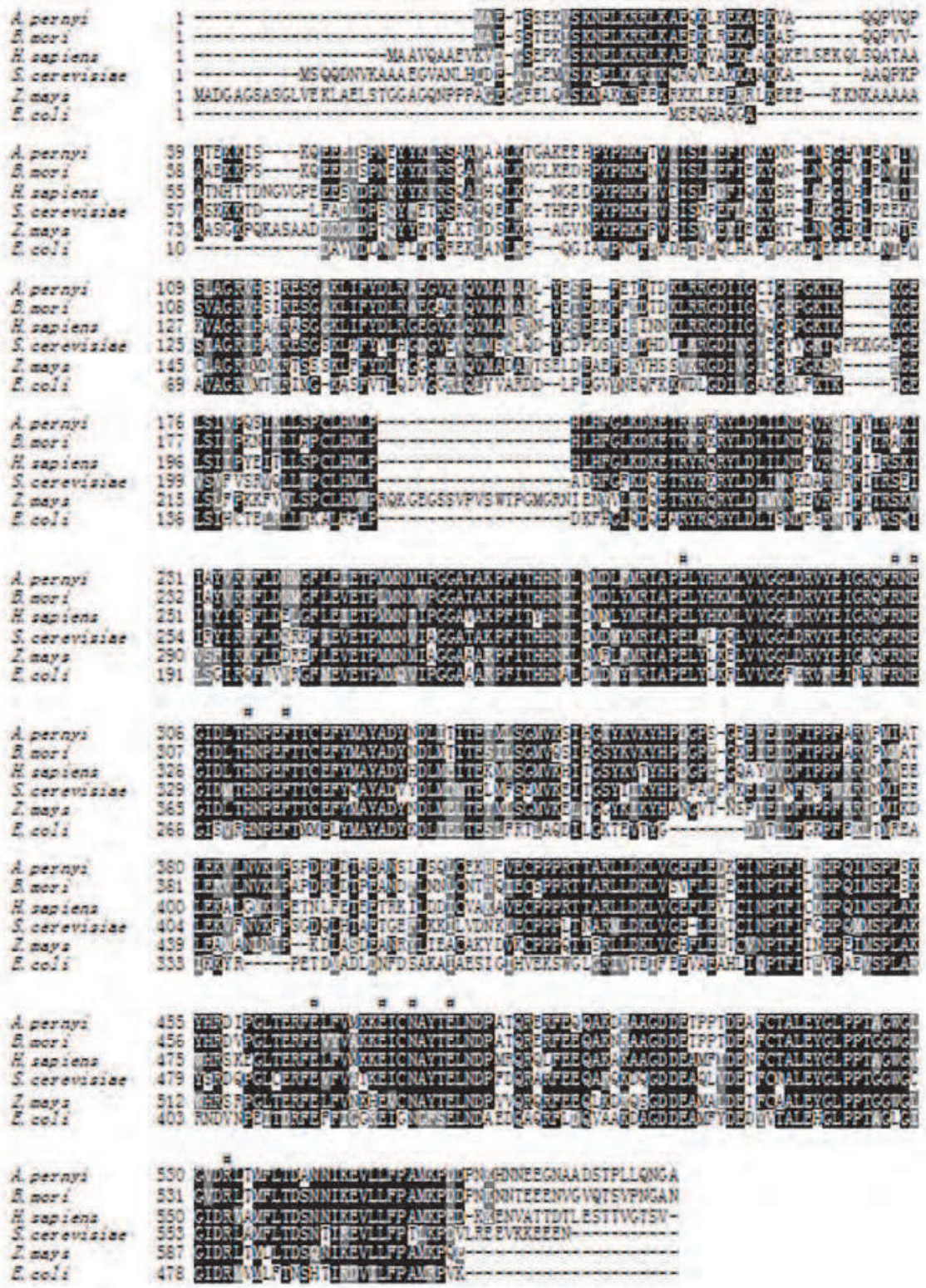

Fig. 2. Sequence alignment of LysRS proteins from $A$. pernyi and other organisms. These LysRs proteins were included from A. pernyi, Bombyx mori (BGIBMGA002984-PA in SilkDB), Drosophila melanogaster (NP_572573), Homo sapiens (NP_005539), Zea mays (NP_001146902), Saccharomyces cerevisiae (AAA6691), Escherichia coli (NP_417366). The number sign (\#) show the residues which form the active site. Identical amino acids are highlighted in black, and positive amino acids are highlighted in gray. 
LysRS revealed 84\% sequence identity to LysRS of Bombyx mori (BGIBMGA002984-PA in SilkDB), $68-74 \%$ to LysRSs of other insects. The A. pernyi LysRS also revealed $57-72 \%$ identity to other invertebrates, $64-71 \%$ identity to vertebrates, $56-59 \%$ identity to plants, and $57 \%$ identity to fungi (Saccharomyces cerevisiae). Note that the A. pernyi LysRS protein showed 37-42\% sequence identity to LysRS of bacteria. In Figure 2, the sequence is shown aligned with six LysRS proteins from A. pernyi, B. mori (BGIBMGA002984-PA in SilkDB), Drosophila melanogaster (NP_572573), H. sapiens (NP_005539), Zea mays (NP_001146902), S. cerevisiae (AAA6691), E. coli (NP_417366). High level of conservation of the amino acid sequence among these LysRS proteins indicates that the LysRS proteins are highly conserved during the evolution of life organisms.

Moreover, by sequence alignment, we found that, the active site residues of LysRS (Desogus et al., 2000), responsible for the ATP-dependent formation of the enzyme bound aminoacyladenylate, are identical among various kinds of organisms including $A$. pernyi collected in this study (Figure 2 and data not shown). This finding suggested that the A. pernyi LysRS is sufficient to have the catalytic activity. The high level of conservation and identical active sites are suggestive of a critical function that these proteins must play in all the organisms where they are found.

\subsection{Phylogenetic analysis}

A total of 43 representative LysRS protein sequences from various organisms including $A$. pernyi were used to reconstruct the phylogenetic relationship. The final alignment resulted in 678 amino acid sites including gaps. Of these sites, 101 were conserved, 562 were variable, and 477 were informative for parsimony. A neighbor-joining tree was constructed using amino acid sequences and a poisson-corrected distance with bootstrap test of 1000 replications (Figure 3). The A. pernyi LysRS protein sequence was found to be closely related to that of $B$. mori with $100 \%$ confidence support. The used LysRS sequences were well divided into five groups corresponding to the known bacteria, fungi, plants, invertebrates and vertebrates.

Within the plant group, three subgroups were well defined corresponding to algae (Chlamydomonas reinhardtii), magnoliopsida (Vitis vinifera) and liliopsida (Z. mays).

Within the vertebrate group, four subgroups were well clustered corresponding to mammalia (H. sapiens), archosauria (Gallus gallus), amphibia (Xenopus laevis) and actinopterygii (Danio rerio). Within the invertebrate group, four subgroups were also well defined corresponding to insects and hard tick (Branchiostoma floridae), cnidaria (Hydra magnipapillata), nematoda (Caenorhabditis elegans). Moreover, within the insect subgroup, all the lepidopterans, dipterans and hymenopterans species were further clearly separated. These results agreed with the topology tree on the classical systematics and other molecular data, such as the will die slowly gene (Li et al., 2011), suggesting the potential value of LysRS protein in phylogenetic inference of life organisms.

\subsection{Expression patterns}

We performed semi-quantitative RT-PCR to detect the quantify the A. pernyi LysRS gene expression levels, by using an actin gene as an internal control that was a constitutively expressed gene (Wu et al, 2010). The results showed that the A. pernyi LysRS gene was expressed during four developmental stages (egg, larva, pupa and adult) (Figure 4), suggesting that the product of the LysRS gene plays an essential role throughout the entire life cycle of $A$. pernyi. 
Molecular Cloning, Expression Pattern, and Phylogenetic

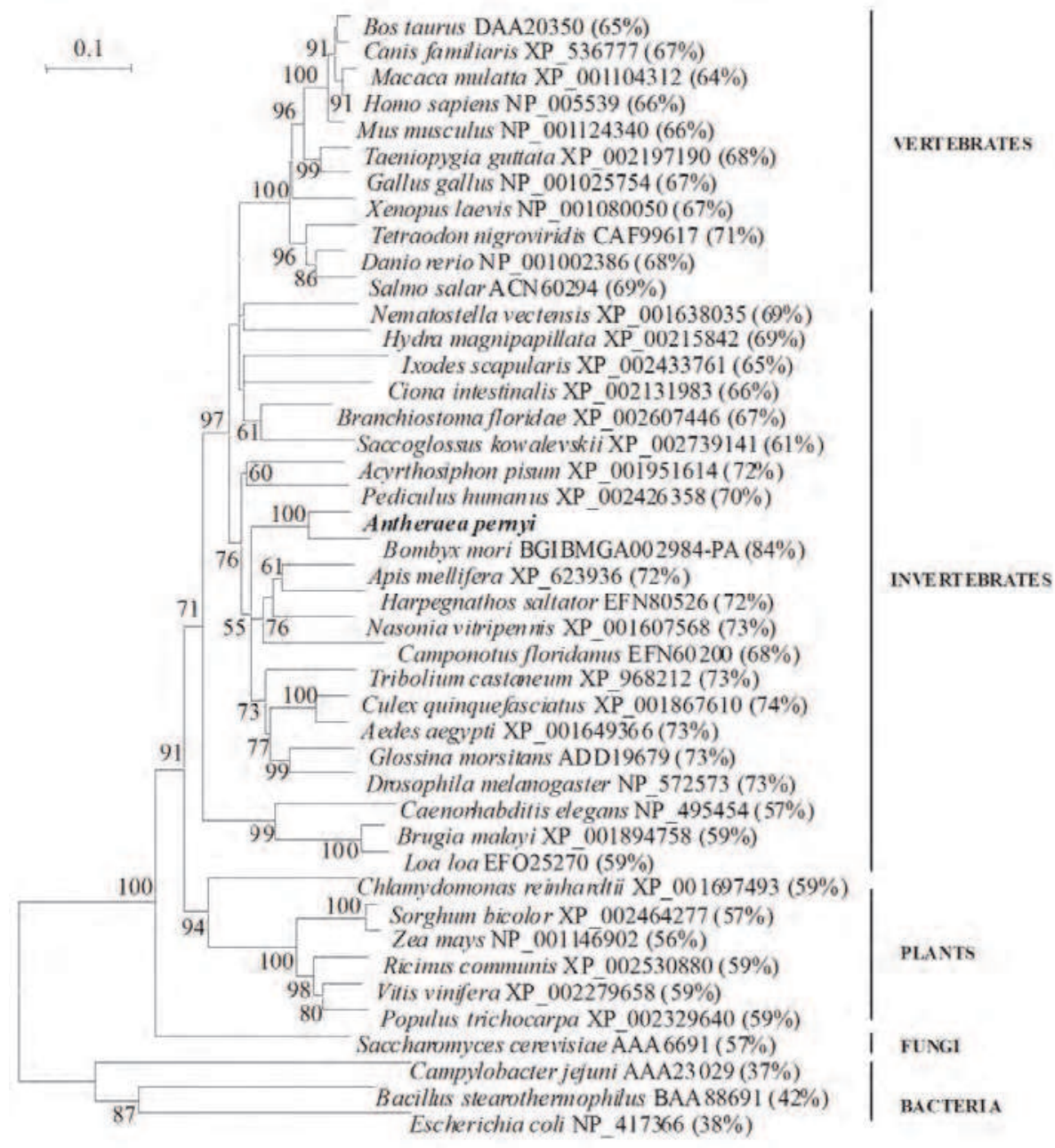

Fig. 3. Phylogenetic tree based on the amino acid sequence comparisons of LysRS proteins from various organisms including $A$. pernyi. Numbers at nodes represent bootstrap P-values $(>50 \%)$. Public database accession numbers of LysRS proteins are shown following the names of organisms. Identity (\%) in parentheses following accession number is obtained by pairwise alignment of amino acid sequence of $A$. pernyi LysRS with indicated LysRSs from other organisms. 


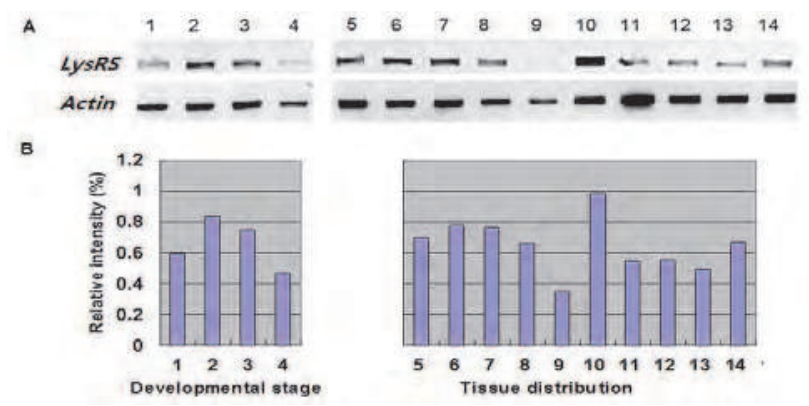

Fig. 4. Expression patterns of the A. pernyi LysRS mRNA in different developmental stages and different tissues of fifth instar larvae preformed by semi-quantitative RT-PCR. RT-PCR was amplified after 30 cycles with specific primer pair for the A. pernyi LysRS gene. The actin gene was used as an internal standard to normalize the templates. Relative expression profiles of A. pernyi LysRS were normalized with actin level. Lanes: 1, eggs at day 5; 2, larvae of fifth instar; 3, pupae; 4, moths; 5, blood; 6, fat body; 7, midgut; 8, silk glands; 9, body wall; 10, Malpighian tublues; 11, spermaries; 12 , ovaries; 13 , brain; 14, muscle. (A) The electrophoretic results. (B) The relatively intensity.

Tissue distributions in fifth instar larvae of the A. pernyi LysRS gene were also analyzed. The results showed that the $A$. pernyi LysRS gene mRNA was present in all tissues tested including blood, fat body, midgut, silk glands, body wall, Malpighian tubules, spermaries, ovaries, brain and muscle (Figure 4). Large-scale EST resource for B. mori, a lepidopteran model insect, are available at GenBank database. The in silico gene expression analysis based on the available EST resources showed the B. mori LysRS gene was also expressed in imaginal disks and pheromone gland not analyzed in this study.

The results also showed that the mRNA levels of the A. pernyi LysRS gene were most abundant in Malpighian tubules. Malpighian tubules system is a type of excretory and osmoregulatory system in insect, including A. pernyi. The system consists of branching tubules extending from the alimentary canal that absorbs solutes, water, and wastes from the surrounding hemolymph. The wastes then are released from the organism in the form of solid nitrogenous compounds. Therefore, the high expression of the gene in the Malpighian tubules corresponds to its role in the development of A. pernyi.

\section{Conclusion}

In the present study, the full length cDNA of the A. pernyi LysRS gene was isolated and characterized from a pupal cDNA library by random EST sequencing. The obtained cDNA sequence consists of 2136 bp nucleotides encoding a polypeptide of 579 amino acids which contains the LysRS class II core domain including the conserved active sites and three characteristic sequence motifs. RT-PCR analysis showed that the A. pernyi LysRS gene was transcribed during four developmental stages (egg, larva, pupa, and moth) and in all the tissues tested (blood, midgut, silk glands, Malpighian tublues, spermaries, ovaries, brain, muscle, fat body and body wall), with most abundance in Malpighian tubules. By searching in database, the A. pernyi LysRS protein homologues were found in various kinds of life organisms, including bacteria, fungi, plants, invertebrates and vertebrates, with $37-84 \%$ amino acid sequence identity, suggesting that they are highly conserved during the 
evolution of life organisms. Phylogenetic analysis based on the LysRS protein homologue sequences clearly separated the known bacteria, fungi, plants, invertebrates and vertebrates, consistent with the topology tree on the classical systematics, suggesting the potential value of LysRS protein in phylogenetic inference of life organisms.

\section{Acknowledgment}

This work was supported by grants from the National Natural Science Foundation of China (No. 31072082), the National Modern Agriculture Industry Technology System Construction Project (Silkworm and Mulberry), the Support Project for Tip-top Young and Middle-aged Talent of Shenyang Agricultural University, the Scientific Research Project for High School of the Educational Department of Liaoning Province (No. 2008643).

\section{References}

Chan, V. L. \& Bingham, H.L. (1992). Lysyl-tRNA synthetase gene of Campylobacter jejuni. Journal of Bacteriology, Vol. 174, No. 3, pp. 695-701, ISSN 0021-9193

Desogus, G.; Todone, F.; Brick, P. \& Onesti, S. (2000). Active site of lysyl-tRNA synthetase: structural studies of the adenylation reaction. Biochemistry, Vol.39, No.29, pp. 84188425, ISSN 0006-2960

Duan, J.; Li, R.Q.; Cheng, D.J.; Fan, W.; Zha, X.F.; Cheng, T.C.; Wu, Y.Q.; Wang, J.; Mita, K.; Xiang, Z.H. \& Xia, Q.Y. (2010). SilkDB v2.0: a platform for silkworm (Bombyx mori) genome biology. Nucleic Acids Research, Vol. 38, Suppl 1, pp. D453-456, ISSN 03051048

Emmerich, R.V. \& Hirshfield, I.N. (1987). Mapping of the constitutive lysyl-tRNA synthetase gene of Escherichia coli K-12. Journal of Bacteriology, Vol. 169, No. 11, pp. 5311-5313, ISSN 0021-9193

Guo, M.; Ignatov, M.; Musier-Forsyth, K.; Schimmel, P. \& Yang, X.L. (2008). Crystal structure of tetrameric form of human lysyl-tRNA synthetase: Implications for multisynthetase complex formation. Proceedings of the National Academy of Sciences, Vol. 105, No. 7, pp. 2331-2336, ISSN 1091-6490Hirshfield, I.N., Bloch, P.L. Van Bogelen, R.A. \& Neidhardt, F.C. (1981). Multiple forms of lysyl-transfer ribonucleic acid synthetase in Escherichia coli. Journal of Bacteriology, Vol. 146, No. 1, pp. 345-351, ISSN 0021-9193

Kepp, O.; Gdoura, A.; Martins, I.; Panaretakis, T.; Schlemmer, F.; Tesniere, A.; Fimia, G.M.; Ciccosanti, F.; Burgevin, A.; Piacentini, M.; Eggleton, P.; Young, P.J.; Zitvogel, L.; van Endert, P. \& Kroemer, G. (2010). Lysyl tRNA synthetase is required for the translocation of calreticulin to the cell surface in immunogenic death. Cell Cycle, Vol. 9, No. 15, pp. 3072-3077, ISSN 1538-4101

Li, Y.P.; Xia, R.X.; Wang, H.; Li, X.S.; Liu, Y.Q.; Wei, Z.J.; Lu, C. \& Xiang, Z.H. (2009). Construction of a full-length cDNA library from Chinese oak silkworm pupa and identification of a KK-42 binding protein gene in relation to pupal-diapause termination. International Journal of Biological Sciences, Vol.5, No.4, pp. 451-457, ISSN 1449-2288

Li, Y.P.; Wang, H.; Xia, R.X.; Wu, S.; Shi, S.L.; Su, J.F.; Liu, Y.Q.; Qin, L. \& Wang, Z,D. (2010). Molecular cloning, expression pattern and phylogenetic analysis of the will die slowly gene from the Chinese oak silkworm, Antheraea pernyi. Molecular Biology 
Reports, DOI: 10.1007/s11033-010-0495-2, ISSN 0301-4851, Available online: http://www.springerlink.com/index/Y0112G15354345L7.pdf

Liu, Y.Q.; Li, Y.; Li, X.S. \& Qin, L. (2010a). The origin and dispersal of the domesticated Chinese oak silkworm, Antheraea pernyi, in China: A reconstruction based on ancient texts. Journal of Insect Science, 10.180, ISSN 1536-2442, Available online: insectscience.org/10.180

Liu, Y.Q.; Li, Y.P.; Wang, H.; Xia, R.X.; Li, X.S.; Wan, H.L.; Qin, L.; Jiang, D.F.; Lu, C. \& Xiang, Z.H. (2010b). cDNA cloning and expression pattern of two enolase genes from the Chinese oak silkworm, Antheraea pernyi. Acta Biochimica et Biophysica Sinica, Vol.42, No.11, pp. 816-826, ISSN 1672-9145

Liu, Y.Q.; Li, Y.P.; Wu, S.; Xia, R.X.; Shi, S.L.; Qin, L.; Lu, C. \& Xiang, Z.H. (2010c). Molecular cloning and expression pattern of a lysophospholipase gene from Antheraea pernyi. Annals of the Entomological Society of America, Vol.103, No.4, pp. 647-653, ISSN 00138746

Park, S.G.; Kim, H.J.; Min, Y.H.; Choi, E.C.; Shin, Y.K.; Park, B.J.; Lee, S.W. \& Kim, S. (2005). Human lysyl-tRNA synthetase is secreted to trigger proinflammatory response. Proceedings of the National Academy of Sciences, Vol. 102, No. 18, pp. 6356-6361, ISSN 1091-6490

Saitou, N. \& Nei, M. (1987). The neighbor-joining method: a new method for reconstructing phylogenetic trees. Molecular Biology E Evolution, Vol. 4, pp. 406-425, ISSN 07374038

Tamura, K.; Dudley, J.; Nei, M. \& Kumar, S. (2007). MEGA4: Molecular Evolutionary Genetics Analysis (MEGA) software version 4.0. Molecular Biology E Evolution, Vol. 24, pp. 1596-1599, ISSN 0737-4038

Thompson, J.D.; Gibson, T.J.; Plewniak, F.; Jeanmougin, F. \& Higgins, D.G. (1997). The CLUSTAL_X windows interface: flexible strategies for multiple sequence alignment aided by quality analysis tools. Nucleic Acids Research, Vol. 25, pp. 4876-4882, ISSN 0305-1048

VanBogelen, R.A.; Vaughn, V. \& Neidhardt, F.C. (1983). Gene for heat-inducible lysyl-tRNA synthetase (lysU) maps near cadA in Escherichia coli. Journal of Bacteriology, Vol. 153, No. 2, pp. 1066-1068, ISSN 0021-9193

Wolf, Y.I.; Aravind, L.; Grishin, N.V. \& Koonin, E.V. (1999). Evolution of aminoacyl-tRNA synthetases--analysis of unique domain architectures and phylogenetic trees reveals a complex history of horizontal gene transfer events. Genome Research, Vol. 9, No. 8, pp. 689-710, ISSN 1474-760X

Wu, S.; Xuan, Z.X.; Li, Y.P.; Li, Q.; Xia, R.X.; Shi, S.L.; Qin, L.; Wang, Z.D. \& Liu, Y.Q. (2010). Cloning and characterization of the first actin gene in Chinese oak silkworm, Antheraea pernyi. African Journal of Agricultural Research, Vol. 5, Nol. 10, pp. 10951100, ISSN 1991-637X 


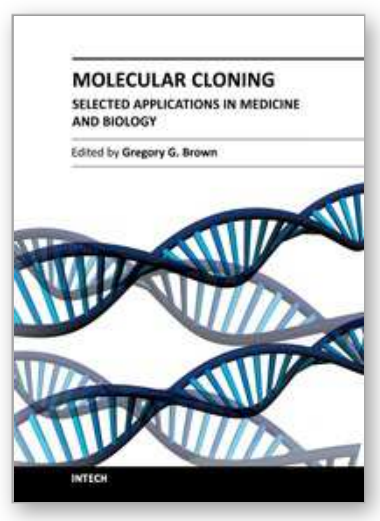

\author{
Molecular Cloning - Selected Applications in Medicine and Biology \\ Edited by Prof. Gregory Brown
}

ISBN 978-953-307-398-9

Hard cover, 324 pages

Publisher InTech

Published online 12, October, 2011

Published in print edition October, 2011

The development of molecular cloning technology in the early 1970 s created a revolution in the biological and biomedical sciences that extends to this day. The contributions in this book provide the reader with a perspective on how pervasive the applications of molecular cloning have become. The contributions are organized in sections based on application, and range from cancer biology and immunology to plant and evolutionary biology. The chapters also cover a wide range of technical approaches, such as positional cloning and cutting edge tools for recombinant protein expression. This book should appeal to many researchers, who should find its information useful for advancing their fields.

\title{
How to reference
}

In order to correctly reference this scholarly work, feel free to copy and paste the following:

Yan-Qun Liu and Li Qin (2011). Molecular Cloning, Expression Pattern, and Phylogenetic Analysis of the LysyltRNA Synthetase Gene from the Chinese Oak Silkworm Antheraea pernyi, Molecular Cloning - Selected Applications in Medicine and Biology, Prof. Gregory Brown (Ed.), ISBN: 978-953-307-398-9, InTech, Available from: http://www.intechopen.com/books/molecular-cloning-selected-applications-in-medicine-andbiology/molecular-cloning-expression-pattern-and-phylogenetic-analysis-of-the-lysyl-trna-synthetase-gene-fro

\section{INTECH}

open science | open minds

\section{InTech Europe}

University Campus STeP Ri

Slavka Krautzeka 83/A

51000 Rijeka, Croatia

Phone: +385 (51) 770447

Fax: +385 (51) 686166

www.intechopen.com

\section{InTech China}

Unit 405, Office Block, Hotel Equatorial Shanghai

No.65, Yan An Road (West), Shanghai, 200040, China

中国上海市延安西路65号上海国际贵都大饭店办公楼 405 单元

Phone: +86-21-62489820

Fax: +86-21-62489821 
(C) 2011 The Author(s). Licensee IntechOpen. This is an open access article distributed under the terms of the Creative Commons Attribution 3.0 License, which permits unrestricted use, distribution, and reproduction in any medium, provided the original work is properly cited. 